\title{
DIFFUSIVE REGIME FOR CRITICAL SOUND ATTENUATION AND DISPERSION IN MAGNETS
}

\author{
A. Pawlak and M. Blaszak \\ Institute of Physics, A. Mickiewicz University \\ Umultowska 85, 61-614 Poznań, Poland
}

\begin{abstract}
We consider a new regime of sound propagation in magnetic systems above the critical point. On the basis of a general statistical model including a flow of energy between spin and lattice subsystems we found that there is a region of parameters where the sound attenuation and dispersion can be expressed in a similar way as for noncritical systems for which only the heat conduction matters. Contrary to the case of the classical heat diffusion in the critical magnetic system both the specific heat, entering the reduced frequency, as well as the effective amplitude are frequency and temperature dependent reflecting the critical singularities present in the system near its critical point.
\end{abstract}

PACS numbers: 05.70.Jk, 62.65.+k

In magnetic insulators, contrary to the metals, critical behaviour of sound attenuation and dispersion is only weakly singular or nonsingular [1]. The phenomenological theory of Kawasaki [2] explained this weakly singular behaviour by postulating that, in insulators, the dominant interaction between the phonon and spin subsystems is of the form of linear coupling of the longitudinal sound mode to the spin-energy density (in metals the coupling of the sound mode to two spin fluctuations should dominate). Recently it was shown $[3,4]$ that it is not the strength of the coupling constant but rather the ratio of relevant relaxation times, which is responsible for the weak singularities. It was also shown that several different regimes may appear in the same critical system, depending on the sound frequency, reduced temperature and relevant relaxation rates. In this paper we present a new regime, similar in some aspects to the classical heat diffusion process, which has not been discussed yet.

The equilibrium probability distribution is determined by the Hamiltonian [4]

$$
\begin{aligned}
H= & \frac{1}{2} \int \mathrm{d}^{d} x\left[r S^{2}+(\nabla S)^{2}+\frac{u}{2} S^{4}+C_{12}\left(\sum_{\alpha} e_{\alpha \alpha}\right)^{2}+2 C_{44} \sum_{\alpha, \beta} e_{\alpha \beta}^{2}\right. \\
& \left.+\frac{e_{\mathrm{S}}^{2}}{C_{\mathrm{S}}}+\frac{e_{\mathrm{L}}^{2}}{C_{\mathrm{L}}}+2 g \sum_{\alpha} e_{\alpha \alpha} S^{2}+2 f e_{\mathrm{S}} S^{2}+2 w\left(e_{\mathrm{S}}+a e_{\mathrm{L}}\right) \sum_{\alpha} e_{\alpha \alpha}\right],
\end{aligned}
$$


where $C_{44}=2\left(C_{11}-C_{12}\right)$, the order-parameter (here the local magnetization) is denoted by $S(x), e_{\alpha \beta}(x)$ are components of the strain tensor, which can be decomposed into normal mode (phonon) variables, and $e_{\mathrm{S}}(x)$ and $e_{\mathrm{L}}(x)$ are spin and lattice energy densities, respectively. The symbols $C_{11}$ and $C_{12}$ stand for the bare elastic constants of isotropic media. The constants $g$ and $w$ describe the coupling of the longitudinal sound mode to two spin fluctuations and to the energy densities of subsystems. $C_{\mathrm{L}}$ and $C_{\mathrm{S}}$ are the bare specific heats.

We define the dynamics of the model by the following Langevin equations:

$$
\begin{aligned}
& \dot{S}_{\boldsymbol{k}}=-\Gamma \frac{\delta H}{\delta S_{-\boldsymbol{k}}}+\xi_{\boldsymbol{k}}, \\
& \ddot{Q}_{\boldsymbol{k}}=-\frac{\delta H}{\delta Q_{-\boldsymbol{k}}}-\theta k^{2} \dot{Q}_{k}+\eta_{\boldsymbol{k}}, \\
& \dot{e}_{\boldsymbol{k}}^{\mathrm{S}}=-\left(\gamma_{\mathrm{S}}+\lambda_{\mathrm{S}} k^{2}\right) \frac{\delta H}{\delta e_{-\boldsymbol{k}}^{\mathrm{S}}}+\gamma \frac{\delta H}{\delta e_{-\boldsymbol{k}}^{\mathrm{L}}}+\varphi_{\boldsymbol{k}}, \\
& \dot{e}_{\boldsymbol{k}}^{\mathrm{L}}=-\left(\gamma_{\mathrm{L}}+\lambda_{\mathrm{L}} k^{2}\right) \frac{\delta H}{\delta e_{-\boldsymbol{k}}^{\mathrm{L}}}+\gamma \frac{\delta H}{\delta e_{-\boldsymbol{k}}^{\mathrm{S}}}+\psi_{k},
\end{aligned}
$$

where $Q_{\boldsymbol{k}}$ is the longitudinal phonon coordinate and the Gaussian white noises $\xi_{\boldsymbol{k}}, \eta_{k}, \varphi_{k}$ and $\psi_{\boldsymbol{k}}$ have zero means and variances related to the bare damping terms through the Einstein relations. The last two equations describe the spin-lattice relaxation as well as the heat conduction in both subsystems. In insulators we can take $\gamma_{\mathrm{L}}=\gamma_{\mathrm{S}}=\gamma$, i.e. the energy in the total spin and lattice system is assumed to be conserved.

The equations of motion are equivalent, in the path-integral formalism, to a Lagrangian [4], on which some decoupling transformations can be performed. We can, for example, decouple the lattice energy field from the others. The parameters in the Lagrangian get renormalised, and instead of the bare spin-energy response function $D_{\mathrm{SO}}(k, \omega)$, we should use a renormalised one

$$
\tilde{D}_{\mathrm{S} 0}^{-1}(k, \omega)=D_{\mathrm{S} 0}^{-1}(k, \omega)-\frac{\gamma^{2} D_{\mathrm{L} 0}}{\Gamma_{\mathrm{S}}(k) C_{\mathrm{S}} \Gamma_{\mathrm{L}}(k)}=C_{\mathrm{S}}^{-1}[m(k)-\mathrm{i} \tilde{\omega} b(\omega)],
$$

where $D_{\mathrm{S} 0}(k, \omega)=\left[-\mathrm{i} \omega / \Gamma_{\mathrm{S}}+C_{\mathrm{S}}^{-1}\right]^{-1}, \mathrm{D}_{\mathrm{L} 0}(\mathrm{k}, \omega)=\left[-\mathrm{i} \omega / \Gamma_{\mathrm{L}}+\mathrm{C}_{\mathrm{L}}^{-1}\right]^{-1}, \Gamma_{\mathrm{i}}(\mathrm{k})=$ $\gamma_{\mathrm{i}}+\lambda_{\mathrm{i}} \mathrm{k}^{2}$ for $i=\mathrm{S}, \mathrm{L}$, and $b(\omega)=\frac{1-\mathrm{i} \bar{\omega}}{m-\mathrm{i} \bar{\omega}}, m(k)=1-\frac{\gamma^{2}}{\Gamma_{\mathrm{S}} \Gamma_{\mathrm{L}}}$ with $\bar{\omega}=\omega C_{\mathrm{L}} / \Gamma_{\mathrm{L}}$ and $\tilde{\omega}=\omega C_{\mathrm{S}} / \Gamma_{\mathrm{S}}$. For the ultrasound wave vectors $m(k) \approx\left(\lambda_{+} k^{2}\right) / \gamma$ is much smaller than unity, where $\lambda_{+}=\lambda_{S}+\lambda_{\mathrm{L}}$ is the total thermal conductivity. The energy response function $\tilde{D}_{\mathrm{S} 0}(k, \omega)$ for high frequencies behaves as $\tilde{D}_{\mathrm{S} 0}(k, \omega) \approx \frac{C_{-}}{1-\mathrm{i} \omega \bar{C}_{-} / \gamma}$, where $C_{-}=\frac{C_{S} C_{L}}{C_{S}+C_{\mathrm{L}}}$ is the heat capacity for the process of equilibration of the lattice and spin temperatures [4]. The function $\tilde{D}_{\mathrm{SO}}$ describes then the process of spin-lattice relaxation with the bare spin-lattice relaxation time $\tau_{\mathrm{SL}}^{0}=C_{-} / \gamma$. For low frequencies $\tilde{D}_{\mathrm{S} 0}$ behaves as

$$
\tilde{D}_{\mathrm{SO}}(k, \omega) \approx \frac{C_{\mathrm{S}}}{1-\frac{\mathrm{i} \omega C_{+}}{\lambda_{+} k^{2}}},
$$


where $C_{+}=C_{\mathrm{S}}+C_{\mathrm{L}}$ is the heat capacity for the processes in which spins and lattice are at equilibrium. The response function $\tilde{D}_{\text {So }}$ describes then the process of heat diffusion assuming that the equilibrium between the spin and lattice subsystems has been reached. The bare thermal conduction rate is given by $\omega_{\text {cond }}=\lambda_{+} k^{2} / C_{+}$.

Applying a number of Gaussian transformations, among which is a transformation decoupling the sound and energy from the order-parameter system, we obtain finally the phonon self-energy

$$
\frac{\Sigma(k, \omega)}{k^{2}}=\frac{2\left[g^{2}(\omega)-\mathrm{i} \tilde{\omega} b(\omega) g^{2}\right] \Psi+w^{2}(\omega) C_{S}}{1-\mathrm{i} \tilde{\omega} b(\omega)(1+v \Psi)}
$$

where $\Psi=\left\langle\Gamma \widetilde{S^{2}}-\boldsymbol{k},-\omega S_{\boldsymbol{k}, \omega}^{2}\right\rangle^{L_{A}}$, with $\widetilde{S^{2}}{ }_{\boldsymbol{k}, \omega}=(\tilde{S} S)_{\boldsymbol{k}, \omega}$, is a four-spin response function evaluated with an effective order-parameter Lagrangian $L_{A} \cdot g(\omega)$ and $w(\omega)$ are effective frequency-dependent coupling constants. The function $\Psi$ has a singularity of the specific-heat type $\Psi=t^{-\alpha} \Phi(y)$, where $\Phi(y)$ is a scaling function, $y=\omega / \omega_{c}$ is the frequency reduced with the characteristic order-parameter rate $\omega_{c}=\Gamma t^{z \nu}$ and $t=\frac{T-T_{C}}{T_{C}}$ is the reduced temperature. The finer characteristics of the energy fields dynamics are contained in the non-universal frequency-dependent coefficient $b(\omega)$, which for $m(k) \ll \bar{\omega}$, applied for not very large wave vectors of the sound wave propagating in an insulator, can be approximated by $i \tilde{\omega} b(\omega)=C_{\mathrm{S}} / C_{\mathrm{L}}-\mathrm{i} \tilde{\omega}(1+$ $\left.m / \bar{\omega}^{2}\right)$. Usually $m / \bar{\omega}^{2} \ll 1$ and this regime was investigated in detail elsewhere [4], however in some favorable cases it may happen that for low frequencies $m / \bar{\omega}^{2} \gg 1$. If, in addition, $m / \bar{\omega}^{2} \gg y$ we obtain a new regime of the critical sound attenuation and dispersion, for which these quantities can be very well approximated by

$$
\begin{aligned}
& \alpha(\omega, t) \approx \frac{\omega C_{\mathrm{L}}}{c_{0}^{3} v C_{\mathrm{S}}} \frac{m}{\bar{\omega}}\left[g \frac{\Delta C_{\mathrm{S}}(t)}{C_{+}(t)}-w f C_{\mathrm{S}} \frac{C_{+}^{a}(t)}{C_{+}(t)}\right]^{2}, \\
& c^{2}(\omega, t)-c^{2} \approx-\frac{2 C_{\mathrm{L}}^{2}}{v C_{\mathrm{S}}} \frac{m^{2}}{\bar{\omega}^{2} C_{+}(t)}\left[g \frac{\Delta C_{\mathrm{S}}(t)}{C_{+}(t)}-w f C_{\mathrm{S}} \frac{C_{+}^{a}(t)}{C_{+}(t)}\right]^{2},
\end{aligned}
$$

where $c^{2}=c_{0}^{2}-2 \frac{\hat{g}^{2}}{v C_{\mathrm{S}}} C_{-}(t)$ is the bare sound velocity, renormalised by the fast process of equilibration of temperatures; $C_{\mathrm{S}}(t)=C_{\mathrm{S}}[1+v \Psi(t, 0)], \Delta C_{\mathrm{S}}(t)=$ $C_{\mathrm{S}}(t)-C_{\mathrm{S}}, C_{+}^{a}(t)=C_{\mathrm{S}}(t)+a C_{\mathrm{L}}, C_{+}(t)=C_{\mathrm{S}}(t)+C_{\mathrm{L}}$ and $\hat{g}=g-w f C_{\mathrm{S}}(1-a)$. In Eqs. (8) and (9) the frequency dependence of $C_{+}(t)$ has been neglected.

Let us recall now that for the process of classical heat conduction in noncritical system the sound attenuation and dispersion are given by

$$
\begin{aligned}
& \alpha(\omega)=W^{2} \frac{\omega}{2 c_{0}^{3}} \operatorname{Im} D(\omega) \propto \omega^{2} C^{0}, \\
& c^{2}(\omega)-c_{0}^{2}=-W^{2} \operatorname{Re} D(\omega) \propto-\omega^{2} C^{-1},
\end{aligned}
$$

where $W^{2}$ is a coupling constant and $D(\omega)=\frac{C}{1-\mathrm{i} \omega C / \lambda k^{2}}$ is a frequency dependent specific heat associated with the process of heat conduction. In Eqs. (10) and (11) the adiabatic sound propagation condition $\omega C / \lambda k^{2} \gg 1$ was assumed. It is interesting that our Eqs. (8) and (9) can also be rewritten in a similar form. In- 
troducing an effective temperature dependent coupling $g_{\mathrm{eff}}(t)=g \Delta C_{\mathrm{S}}(t) / C_{+}(t)-$ $w f C_{S} C_{+}^{a}(t) / C_{+}(t)$ and a frequency and temperature dependent specific heat relevant for the process of heat diffusion $C_{+}(t, \omega)=\frac{C_{+}(t)}{1-\mathrm{i} \omega C_{+}(t) / \lambda_{+} k^{2}}$, we obtain

$$
\begin{aligned}
& \alpha(\omega, t) \approx \frac{\omega g_{\mathrm{eff}}^{2}(t)}{c_{0}^{3} v C_{\mathrm{S}}} \operatorname{Im} C_{+}(t, \omega), \\
& c^{2}(\omega, t)-c^{2} \approx-\frac{2 g_{\mathrm{eff}}^{2}(t)}{v C_{\mathrm{S}}} \operatorname{Re} C_{+}(t, \omega),
\end{aligned}
$$

where the adiabatic sound propagation condition is also used. It is seen that the coupling constants $g$ and $w$ have different "weights": $\Delta C_{\mathrm{S}}(t) / C_{+}(t)$ and $C_{+}^{a}(t) / C_{+}(t)$, respectively, in the effective coupling $g_{\text {eff }}(t)$.

The critical singularities are contained in $C_{+}(t)$ and $g_{\text {eff }}(t)$. If $C_{\mathrm{L}} \ll C_{\mathrm{S}}(t)$ then $g_{\text {eff }}(t) \approx$ const and we get

$$
\begin{aligned}
& \alpha(\omega, t) \propto \omega^{2} t^{0}, \\
& c^{2}(\omega, t)-c^{2} \propto-\omega^{2} t^{\alpha} .
\end{aligned}
$$

If, however, $C_{\mathrm{L}} \gg C_{\mathrm{S}}(t)$ and additionally $g \gg a w f C_{\mathrm{S}}$, then $g_{\text {eff }}(t) \propto t^{-2 \alpha}$ and

$$
\begin{aligned}
& \alpha(\omega, t) \propto \omega^{2} t^{-2 \alpha}, \\
& c^{2}(\omega, t)-c^{2} \propto-\omega^{2} t^{-2 \alpha} .
\end{aligned}
$$

For $g \ll a w f C_{S}$ we get non-singular behaviour of both attenuation and dispersion in this case. Equations (14)-(17) show that only weakly singular behaviour of sound attenuation and dispersion is possible in this "diffusive" regime as expected for magnetic insulators [1]. However, more precise ultrasonic measurements are necessary to distinguish between different kinds of these weak singularities.

\section{References}

[1] B. Lüthi, in: Dynamical Properties of Solids, Eds. G.K. Horton, A.A. Marudin, Vol. 3, North-Holland, Amsterdam 1980, p. 245.

[2] K. Kawasaki, Phys. Lett. A 29, 406 (1968).

[3] A. Pawlak, Eur. Phys. J. B 4, 179 (1998).

[4] A. Pawlak, preprint available at: http://xxx.lanl.gov/cond- mat/9903229. 